\title{
Gemcitabine and Cisplatin for patients with metastatic or recurrent esophageal carcinoma: A Southwest Oncology Group study
}

\author{
Susan G. Urba ${ }^{1}$, Kari Chansky², Peter J. vanVeldhuizen ${ }^{3}$, Robert E. Pluenneke ${ }^{4}$, Jacqueline K. \\ Benedetti $^{2}$, John S. Macdonald ${ }^{5}$ and James L. Abbruzzese ${ }^{6}$ \\ ${ }^{1}$ University of Michigan Medical Center, Ann Arbor, MI; ${ }^{2}$ Southwest Oncology Group Statistical Center, Seattle, \\ WA; ${ }^{3}$ University of Kansas Medical Center, Kansas City, MO; ${ }^{4}$ Kansas City Community Clinical Oncology \\ Program, Kansas City, MO; ${ }^{5}$ St Vincent's Comprehensive Cancer Center, New York, NY; ${ }^{6} \mathrm{MD}$ Anderson Cancer \\ Center, Houston, TX, U.S.A.
}

Key words: esophageal cancer, chemotherapy, gemcitabine, cisplatin

\begin{abstract}
Summary
Purpose: Experimental data, both in vivo and in vitro, suggest that the combination of gemcitabine and cisplatin acts synergistically. Within the Southwest Oncology Group, we designed a Phase II trial to test this chemotherapy combination for patients with esophageal cancer. Experimental design: Patients with metastatic or recurrent esophageal cancer were treated with gemcitabine $1000 \mathrm{mg} / \mathrm{m}^{2}$ on days 1,8 , and 15 , and cisplatin $100 \mathrm{mg} / \mathrm{m}^{2}$ on day 15. Cycles were repeated every 28 days. The statistical endpoint was overall survival. Results: Sixty-four eligible patients were accrued from 37 institutions. Twenty-six percent of patients had prior chemotherapy. The treatment was generally well-tolerated, with the most common toxicity being neutropenia in $31 \%$ of patients. All 64 patients have died. Survival at 3 months was $81 \%$, and at 1 year was $20 \%$. Median survival was 7.3 months. Conclusions: This regimen is tolerable palliative option for patients with metastatic esophageal cancer.
\end{abstract}

\section{Introduction}

Patients with metastatic or recurrent esophageal cancer have a very poor prognosis. Chemotherapy is not able to cure this advanced disease, and it cannot predictably prolong survival. The rationale for administering chemotherapy to patients with metastatic disease is palliation of distressing symptoms.

Relatively few chemotherapy agents are effective against esophageal cancer, and therefore any new agents with possible activity in this disease warrant exploration. Typical chemotherapy agents for this disease and their response rates as single agents include cisplatin - 20\% [1], 5 - fluorouracil - $16 \%$ [2], paclitaxel - 32\% [3], and mitomycin-C - 18\%$30 \%$ [4], irinotecan $-33 \%$ in gastric cancer (5), and docetaxel $-18 \%$ in chemotherapy-naïve patients [6]. Combination chemotherapy yields slightly higher response rates.
Unfortunately, a good response rate does not necessarily translate into improved survival. The median survival of patients diagnosed with metastatic disease is 4-8 months [7]. In the most recent experience of the Southwest Oncology Group utilizing a Phase II agent, the estimated median survival in this population was 3 months [8].

Gemcitabine (difluorodeoxycytidine), an analog of cytosine arabinoside (ara-C), is a pyrimidine antimetabolite [9]. The mechanism of action of gemcitabine has been well characterized. Gemcitabine is activated by deoxycytidine kinase to difluorodeoxycytidine monophosphate (dFdCMP). Then, dFdCMP is further metabolized to difluorodeoxycytidine diphosphate (dFdCDP) and triphosphate (dFdCTP) which, when incorporated into DNA, results in chain termination. In comparison to ara-C incorporation into DNA, $\mathrm{dFdCTP}$ is less readily excised from DNA by DNA exonuclease. Thus, dFdCTP accumulates 
intracellularly to a greater degree than ara-C. This may account, in part, for its different spectrum of preclinical and clinical activity. In addition, gemcitabine inhibits ribonucleotide reductase, an enzyme that produces deoxynucleotides that are required for DNA synthesis.

The favorable toxicity profile of gemcitabine does not overlap with that of cisplatin, and the two drugs have differing mechanisms of action as well. Cisplatin is a cycle-specific agent that acts by binding to DNA and produces DNA crosslinks. Gemcitabine is a phase-specific agent that inhibits excision repair of cisplatin-damaged DNA [10].

Experimental data, both in vitro and in vivo, suggest that the gemcitabine-cisplatin combination should act synergistically. This effect is dependent on the schedule of administration. Synergism was observed in a wild-type cell line and in a cisplatinresistant cell line, but not in a gemcitabine-resistant cell line. It is likely that previous incorporation of gemcitabine to a certain extent into DNA is necessary to achieve an interaction with cisplatin, and that the synergism that occurs is largely because of inhibition of cisplatin-induced DNA repair [11,12].

Based on this potential synergism, we designed a Phase II trial of cisplatin and gemcitabine for patients with metastatic or recurrent esophageal cancer, conducted within the Southwest Oncology Group (S9801). A previous Southwest Oncology Group trial in this disease site yielded median survival of 3 months. Although response has been the traditional standard for evaluation of Phase II agents, it is a difficult endpoint to accurately assess in diseases with poor survival, due to rapid patient deterioration prior to planned timing of scans, and due to increased reluctance of institutions to schedule expensive scans. Thus, in recent years the Southwest Oncology Group has begun designing Phase II trials based on survival endpoints. For this study, the primary objectives were to assess overall survival in these patients, and to assess the toxicities of this regimen.

\section{Methods \\ Eligibility criteria}

Patients were required to have biopsy-proven squamous cell carcinoma or adenocarcinoma of the esophagus or GE junction. Eligible patients included those who were newly diagnosed with metastatic disease, or those with local or metastatic recurrence of disease. Patients could have measurable or evaluable disease. Patients could not have had prior treatment for metastatic or recurrent disease; they were allowed to have had prior chemotherapy or radiation if administered in the neoadjuvant setting at initial diagnosis. If previously treated, there had to be at least a 3month interval between the last day of cisplatin and registration to this study. Patients were not allowed to have received prior gemcitabine. At least 28 days must have elapsed since completion of any other previous treatment. Southwest Oncology Group Performance Status of 0-2 was required, and the following pretreatment laboratory parameters had to be fulfilled: granulocyte count $>1500 / \mu$ l, platelets $>100,000 / \mu 1$, serum creatinine $<2 \times$ the institutional upper limit of normal, a creatinine clearance $>60 \mathrm{ml} / \mathrm{min}$, bilirubin $<2 \times$ institutional upper limit of normal, and SGOT or SGPT $<2.5 \times$ institutional upper limit of normal, or $<5 \times$ institutional upper limit of normal if the liver was involved with tumor. No prior malignancies were allowed except for the following: adequately treated basal cell or squamous cell skin cancer, adequately treated Stage I or II cancer from which the patient is currently in complete remission, or any other cancer from which the patient has been disease-free for 5 years. Patients could not have serious concomitant systemic disorders or active central nervous system metastases. Patients were informed of the investigational nature of the study and signed written informed consent in accordance with institutional and federal guidelines.

\section{Treatment plan}

Gemcitabine $1000 \mathrm{mg} / \mathrm{m}^{2}$ IV over $30 \mathrm{~min}$ was administered on days 1,8 , and 15 , followed by a 1 -week rest. Cycles were given q 28 days. Cisplatin $100 \mathrm{mg} / \mathrm{m}^{2}$ IV over $90 \mathrm{~min}$ was administered on day 15 of each 28-day cycle, after the gemcitabine had been given. Appropriate antiemetics were given prior to each chemotherapy treatment. Prehydration for the cisplatin was $1500 \mathrm{cc}$ of normal saline plus $20 \mathrm{mEq}$ potassium chloride followed by $12.5 \mathrm{mEq}$ mannitol IV. push. Then, the cisplatin was administered, followed by $1000 \mathrm{cc}$ normal saline.

The protocol specified dose modifications in the event the patient should experience a specific toxicity. Dosage modifications were dictated if a patient experienced any of the following hematologic toxicities: if the absolute granulocyte count nadir was 1000-1499 or if the platelet nadir was 
$50,000-99,999$, the gemcitabine dose was changed to $500 \mathrm{mg} / \mathrm{m}^{2}$ and the cisplatin dose to $50 \mathrm{mg} / \mathrm{m}^{2}$; if the granulocyte nadir was 0-999 or if the platelet nadir was $<50,000$, no chemotherapy was given until the granulocyte count returned to $>1000$ and the platelets were $>50,000$, and then the patients were treated at the half/dose level. If the blood counts had not recovered sufficiently after holding treatment for two consecutive weeks, the patient was removed from protocol treatment. If patients completed a 3-week cycle at full doses without nonhematologic toxicity $>$ grade 1, and if absolute granulocyte count was always greater than 1500 and platelets were always greater than 100,000 , then the gemcitabine dose was escalated by $25 \%$.

Modifications were also dictated for nonhematologic toxicities. On the day of scheduled treatment with cisplatin, patients received full dose if the creatinine clearance was $>60 \mathrm{ml} / \mathrm{min}$, or $50 \mathrm{mg} / \mathrm{m}^{2}$ if the creatinine clearance was $40-59 \mathrm{ml} / \mathrm{min}$, or received no further treatment if the creatinine clearance was $<40 \mathrm{ml} / \mathrm{min}$. Cisplatin was decreased to $50 \mathrm{mg} / \mathrm{m}^{2}$ for grade 2 peripheral neuropathy, and discontinued for grade 3 neuropathy. The cisplatin was decreased to $75 \mathrm{mg} / \mathrm{m}^{2}$ for Grade 3-4 nausea and vomiting.

Criteria for removal from the protocol treatment were progression of disease, unacceptable toxicity, delay of greater than 2 weeks beyond the planned treatment date, or the patient's request.

\section{Statistical considerations}

The primary objective of this study was to evaluate the 3-month survival rate in patients with esophageal cancer treated with gemcitabine and cisplatin. Based on the experience of the Southwest Oncology Group in SWOG 9339, in which patients with metastatic esophageal cancer were treated with topotecan $1.5 \mathrm{mg} / \mathrm{m}^{2}$ weekly $\times 4$, every 6 weeks, the estimated median survival in this population was 3 months [8]. It was assumed that the gemcitabine/cisplatin regimen would be of interest if the 3-month survival were $70 \%$ or better. Median survival and the 3-month survival rate were estimated using the Kaplan-Meier method.

\section{Results}

\section{Patient characteristics}

The Southwest Oncology Group initiated this trial on April 1, 1998. Between that date and June 15, 1999,
Table 1. Patient characteristics

\begin{tabular}{|c|c|}
\hline No. of eligible patients & 64 \\
\hline \multicolumn{2}{|l|}{ Age } \\
\hline Median & 57 \\
\hline Range & $33-77$ \\
\hline \multicolumn{2}{|l|}{ Sex } \\
\hline Males & $61(95 \%)$ \\
\hline Females & $3(5 \%)$ \\
\hline \multicolumn{2}{|l|}{ Histology } \\
\hline Adenocarcinoma & $52(81 \%)$ \\
\hline Squamous cell & $10(16 \%)$ \\
\hline Other & $2(3 \%)$ \\
\hline \multicolumn{2}{|l|}{ Disease Status } \\
\hline Metastatic & $29(45 \%)$ \\
\hline Recurrent & $35(55 \%)$ \\
\hline \multicolumn{2}{|l|}{ Prior Therapy } \\
\hline Chemotherapy & 17 \\
\hline Platinum-based & 16 \\
\hline Not specified & 1 \\
\hline Radiation & 19 \\
\hline Surgery & 22 \\
\hline None & 6 \\
\hline \multicolumn{2}{|c|}{ SWOG performance status } \\
\hline 0 & $30(47 \%)$ \\
\hline 1 & $29(45 \%)$ \\
\hline 2 & $5(8 \%)$ \\
\hline
\end{tabular}

73 patients were accrued from 37 institutions. Nine patients were ineligible. Reasons for ineligibility were: inadequate documentation of baseline eligibility requirements (five patients), radiation therapy to a metastatic site prior to study entry (one patient), absence of metastatic or recurrent disease (two patients), and inadequate creatinine clearance (one patient). Patient characteristics for the 64 eligible patients are described in Table 1. Sixty-one patients were male, and the median age was 57. Fifty-two patients $(81 \%)$ had adenocarcinoma, ten patients (16\%) had squamous cell carcinoma, and two patients (3\%) had other pathology (adenosquamous, and undifferentiated). Twenty-nine patients had newly diagnosed metastatic disease, and thirty-five had recurrent disease. Thirty-two patients had measurable disease, and thirty-two had nonmeasurable disease. Seventeen points (26\%) had prior chemotherapy, nineteen $(30 \%)$ had prior radiation, and twenty-two $(35 \%)$ had prior surgery. The prior chemotherapy regimens used were: cisplatin/5-fluorouracil 11 patients; carboplatin/paclitaxel - 3 patients; cisplatin/ paclitaxel - 1 patient; carboplatin/5-fluorouracil/paclitaxel - 1 patient; and unknown - 1 patient. 
Thirty patients (47\%) had a performance status of 0,29 $(45 \%)$ had a performance status of 1 , and $5(8 \%)$ had a performance status of 2 .

\section{Toxicity}

Patients received the following total cycles of chemotherapy: 1-2 cycles -36 patients; $3-4$ cycles - 14 patients; $5-6$ cycles -12 patients; $7-8$ cycles -1 patient, and $>8$ cycles -1 patient.

Thirty-seven patients required a reduction of the dose of gemcitabine, and four patients required a reduction in cisplatin. The primary reason for the dose reductions was neutropenia. However, none of the patients experienced febrile neutropenia, or required hospitalization. In virtually every case the granulocytes recovered within a short period of time without sequelae. Two patients were given a dose escalation of the gemcitabine.

Patients came off of protocol treatment for the following reasons: toxicity - eight patients (four nausea/vomiting, one - tinnitus, one - hearing loss, one - nephrotoxicity, and one - weight loss); refusal eight patients; progression of disease - thirty-seven patients; death while on active treatment - six patients (described below); and other reasons - five patients (two patients had medical problems not related to chemotherapy which delayed treatment $>2$ weeks; one patient had stable disease but no symptom relief and wanted other treatment; one patient had an excellent response and so his physician switched him to more aggressive chemoradiation; and one patient unknown.) There were no major protocol deviations.

Six deaths occurred during active treatment. One was considered definitely related to treatment: renal failure likely related to the cisplatin. One death was possibly related to treatment: a patient with a history of cardiac disease and hypertension had a cardiac arrest 5 days after his first chemotherapy treatment (no neutropenia). Four deaths were considered to be unrelated to the treatment: three were due to disease progression, and one occurred as a complication of an ERCP done to remove a biliary stent in a patient who was clinically improving on chemotherapy.

Toxicities are summarized in Table 2 . The most common grade 3 or 4 toxicities that patients developed were: neutropenia $-31 \%$, leukopenia $-23 \%$, nausea $17 \%$, vomiting - $11 \%$, thrombocytopenia $11 \%$, anemia $-11 \%$, fatigue $-8 \%$, and dehydration $-8 \%$.
Table 2. Chemotherapy toxicity

\begin{tabular}{ll}
\hline Toxicity & Grade 3/4 (\%) \\
\hline Neutropenia & 31 \\
Leukopenia & 23 \\
Nausea & 17 \\
Vomiting & 11 \\
Thrombocytopenia & 11 \\
Anemia & 11 \\
Fatigue & 8 \\
Dehydration & 8 \\
\hline
\end{tabular}

One death due to renal failure, related to treatment; One sudden death, possibly related to treatment; Eight patients discontinued treatment due to toxicity.

\section{Survival}

All 64 patients have died, and have been included in the survival analysis. Median survival is 7.3 months (95\% CI 5.1-8.8). Survival at 3 months is $81 \%$ (95\% CI $72-91$ ) and at 1 year is $20 \%$ (95\% CI $10-30$ ). See Figure 1.

Median time to treatment failure (disease progression or discontinuation of treatment for any reason) was 2.5 months, with $28 \%$ still failure-free at 4 months.

\section{Discussion}

Treatment of metastatic or recurrent esophageal cancer generally begins with a discussion of the possible goals of therapy. Patients must be told that the disease is not curable, and that chemotherapy does not predictably prolong life. It is most commonly used with palliative intent.

Relatively few chemotherapy agents are effective against esophageal cancer, and therefore any new agent with possible activity in this disease warrants exploration. The most commonly used agents have been cisplatin, 5-fluorouracil, mitomycin-C, paclitaxel, and more recently irinotecan, and to some extent docetaxel.

The combination of cisplatin and 5-fluorouracil is one of the most highly used palliative regimens, yielding a response rate of about $35 \%$, and median survival of 6-8 months [13]. However, the numerous side effects of cisplatin (nausea, ototoxicity, nephrotoxicity, and myelosuppression) and 5-fluorouracil (mucositis and diarrhea) limit their usefulness in debilitated patients. 


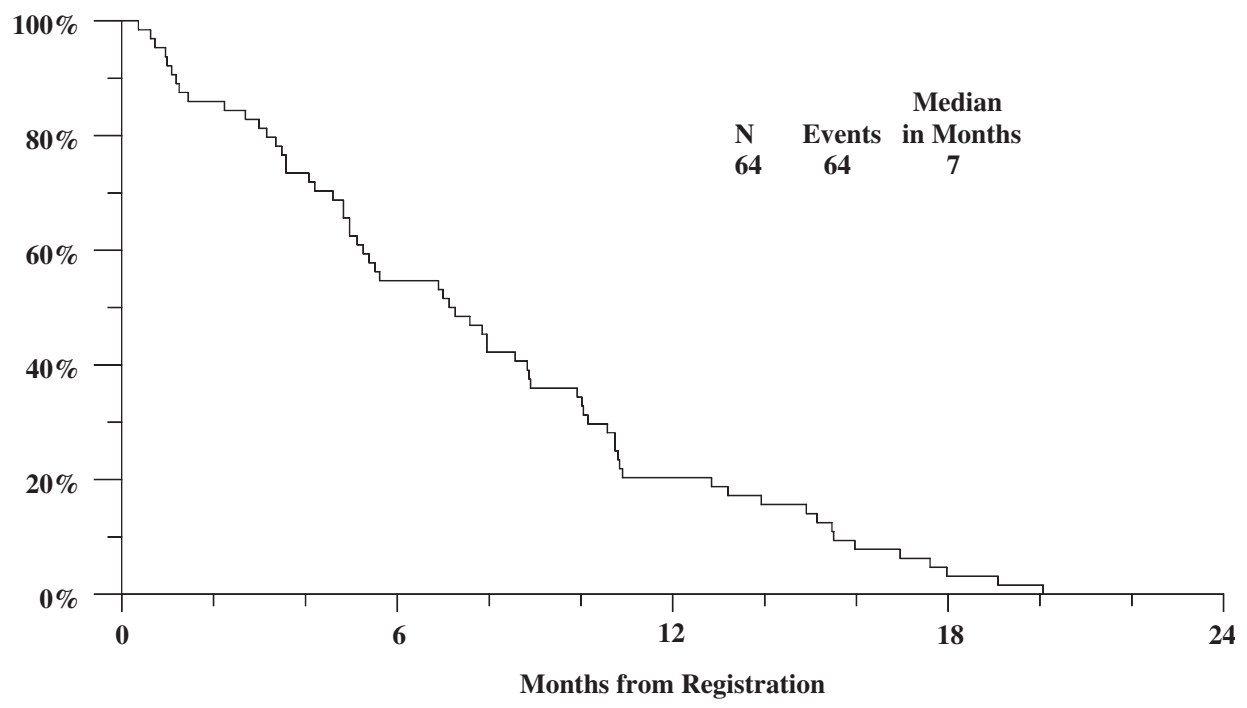

Figure 1. Survival.

Cisplatin and paclitaxel have been combined in Phase I studies, resulting in a response rate of approximately $50 \%[14,15]$. Survival data was not available in these trials. When 5-fluorouracil was added to the cisplatin/paclitaxel combination for 61 patients with esophageal cancer, major responses were seen in $48 \%$ of patients [16]. Median survival was 10.8 months. Toxicity was considered severe but manageable, with $82 \%$ of patients experiencing grade 3 or 4 toxicities. Therefore, the same authors removed the 5-fluorouracil from the combination, and conducted a Phase II trial for 38 chemo-naïve patients with metastatic esophageal cancer [17]. Response rate was $44 \%$ and median survival was 6.9 months. However, there were four treatment-related deaths from this aggressive regimen (paclitaxel $250 \mathrm{mg} / \mathrm{m}^{2}$ later reduced to $200 \mathrm{mg} / \mathrm{m}^{2}$, and cisplatin $75 \mathrm{mg} / \mathrm{m}^{2}$ ).

Gemcitabine is a relatively new agent currently undergoing evaluation for its potential activity in esophageal cancer. Single-agent gemcitabine was administered to 21 patients with chemotherapynaïve metastatic esophageal cancer [18]. The drug was administered at a dose of $1250 \mathrm{mg} / \mathrm{m}^{2}$ over $30-60 \mathrm{~min}$ on days 1,8 , and 15 , followed by 1 week of rest, up to a maximum of six cycles. Grade $3 / 4$ toxicities in 19 evaluable patients were granulocytopenia $-21 \%$, and anemia $-10 \%$. No responses were seen in 17 evaluable patients. Therefore, because of the previously mentioned synergism observed in vitro between gemcitabine and other agents, the possible role for gemcitabine in esophageal cancer may be in combination therapy.

Some trials have been conducted using gemcitabine in combination with other chemotherapy agents. Early data has been reported in abstract form on the combination of gemcitabine and 5-fluorouracil [19]. A Phase II trial was designed based on preclinical evidence of sequence-dependent synergy with this combination. Twenty-three patients with metastatic or advanced esophageal cancer were treated with gemcitabine $1000 \mathrm{mg} / \mathrm{m}^{2}$, 5-fluorouracil $500 \mathrm{mg} / \mathrm{m}^{2}$, and leucovorin $20 \mathrm{mg} / \mathrm{m}^{2}$ on days 1,8 , and 15 of a 28-day cycle. Prior chemotherapy was allowed. Toxicity was mild. Thirty percent of patients experienced grade 3 hematologic toxicity. Sixty percent developed non hematologic grade 3 toxicities, such as diarrhea, nausea, vomiting, infection, and stomatitis, much of which was probably related to the 5-fluorouracil. The response rate in 22 evaluable patients was $32 \%$. Although the trial is small, the early results are promising.

Gemcitabine has also been combined with paclitaxel in a Phase I trial for solid malignancies [20]. Eighteen patients with stage IV malignancies were treated with paclitaxel $150 \mathrm{mg} / \mathrm{m}^{2}$ over 3 h every 21 days, and gemcitabine was given on days 1 and 8 , in three separate dose-escalating cohorts $\left(800,900\right.$, and $1000 \mathrm{mg} / \mathrm{m}^{2}$ over $15 \mathrm{~min}$ ). Two of the patients had esophageal cancer. The dose-limiting toxicity was neutropenia, and the recommended starting dose for future trials was gemcitabine $900 \mathrm{mg} / \mathrm{m}^{2}$. Four of the 
patients (22\%) had an objective response, all of whom had either transitional cell or squamous cell carcinoma.

Sequence-dependent pharmacokinetic and pharmacodynamic interactions have been observed for the combination of gemcitabine and cisplatin [21]. Forty-one patients with a variety of solid tumors were treated with one of four schedules: gemcitabine $800 \mathrm{mg} / \mathrm{m}^{2}$ on days 1,8 , and 15 administered either 4 or $24 \mathrm{~h}$ before cisplatin $50 \mathrm{mg} / \mathrm{m}^{2}$ on days 1 and 8 , or cisplatin administered either 4 or $24 \mathrm{~h}$ before gemcitabine. Cycles were repeated every 28 days. In the second cycle, the sequence was reversed, so that the patient served as his or her own control regarding sequence-related toxicity. The extent of leukopenia was schedule-dependent, with significantly less toxicity occurring when gemcitabine was administered before the cisplatin. Interestingly, of the five patients with esophageal cancer, one patient achieved a complete response, and two patients achieved a partial response. The mean duration of response for these patients was six months.

In our study, half of the patients required a reduction in the dose of gemcitabine, primarily because of neutropenia. However, since no patients developed neutropenic fever or required hospitalization, in retrospect we think that the dose-reduction guidelines could have been less stringent (20\% reduction instead of $50 \%$ ). In this way, a higher overall dose of the gemcitabine could have been delivered. Secondly, the starting dose of cisplatin should have been lower (perhaps 60 or $75 \mathrm{mg} / \mathrm{m}^{2}$ instead of $100 \mathrm{mg} / \mathrm{m}^{2}$ ) since most of the toxicities that caused patients to be removed from the study were related to the cisplatin rather than to the gemcitabine (nausea, vomiting, ototoxicity, and nephrotoxicity.) One of the deaths was definitely related to the cisplatin, and another one was possibly related. Eight patients discontinued treatment due to toxicity, and perhaps this could have been avoided with a lower dose of cisplatin. Some of the patients had been pretreated with cisplatin or radiation before entry into the study, and they may have been able to better tolerate a more "palliative" regimen with less cisplatin, and yet possibly more gemcitabine.

In this trial, we defined the primary survival endpoint in terms of 3-month survival, since our previous trial for patients with metastatic esophageal cancer and similar eligibility criteria yielded only 3 -month median survival. We determined a priori that the regimen would be of further interest if the data were consistent with a true 3-month survival probability of $70 \%$ or greater. Eighty-one percent of patients survived 3 months, with median survival of 7 months.

The combination of gemcitabine and cisplatin was quite tolerable, and some of the toxicity could most likely be eliminated with a slight reduction of the cisplatin dose. Median survival was favorable compared to our last protocol for metastatic disease. Future directions in the Southwest Oncology Group include an ongoing trial of gemcitabine and irinotecan in patients with metastatic disease. Because of gemcitabine's dramatic ability to effect radiationsensitization, the group is also considering a trial of chemoradiation with gemcitabine for patients with locally unresectable disease.

\section{Acknowledgements}

This investigation was supported in part by the following PHS Cooperative Agreement grant numbers awarded by the National Cancer Institute, DHHS: CA38926, CA32102, CA27057, CA12644, CA35176, CA35261, CA35178, CA58882, CA58723, CA22433, CA45807, CA35090, CA52386, CA46113, CA63850, CA58861, CA35192, CA67663, CA37981, CA42777, CA35431, CA13612, CA45450, CA04919, CA46441, CA35262, CA63844, CA46136, CA35281, CA58686, CA20319, CA58415, CA76132, CA76462, and supported in part by Eli Lily.

\section{References}

1. Panettiere FJ, Leichman LP, Tilchen E, Chen TT: Chemotherapy for advanced epidermoid carcinoma of the esophagus with single-agent cisplatin: Final report on a Southwest Oncology Group study. Cancer Treat Rep 68: 1023-1024, 1984

2. Ezdinli EZ, Gelber R, Desai DV, Falkson G, Moertel CG, Han RG: Chemotherapy of advanced esophageal carcinoma: Eastern Cooperative Oncology Group Experience. Cancer 46: 2149-2153, 1980

3. Ajani JA, Ilson DH, Daugherty K, Pazdur R, Lynch PM, Kelsen DP: Activity of taxol in patients with squamous cell carcinoma and adenocarcinoma of the esophagus. J Natl Cancer Inst 86: 1086-1091, 1994

4. Coia L: The use of Mitomycin-C in esophageal cancer. Oncol (suppl) 50: 53-62, 1993

5. Kambe M, Wakui A, Nakao I, Futatsuki K, Sakata Y, Yoshino M, Shimada Y, Taguchi T: A late phase II study of 
irinotecan (CPT-11) in patients with advanced gastric cancers. Proc Am Soc Clin Oncol 12: 584, 1993

6. Heath EI, Urba S, Marshall J, Piantadosi S, Forastiere AA Phase II trial of docetaxel chemotherapy in patients with incurable adenocarcinoma of the esophagus. Invest New Drugs 20: 95-99, 2002

7. Ilson DH, Saltz L, Enzinger P, Huang Y, Kornblith A, Gollub M, O’Reilly E, Schwartz G, DeGroff J, Gonzalez G, Kelsen D: Phase II trial of weekly irinotecan plus cisplatin in advanced esophageal cancer. J Clin Oncol 17: 3270-3275, 1999

8. Macdonald JS, Jacobsen JL, Ketchel SJ, Weiss G, Taylor S, Mills G, Kuebler JP, Rivkin S, Conrad M: A phase II trial of topotecan in esophageal cancer: A Southwest Oncology Group study (SWOG 9339). Invest New Drugs 18: 199-202, 2000

9. Hertel LW, Boder GB, Kroin JS, Rinzel SM, Poore GA Todd GC, Grindey GB: Evaluation of the antitumor activity of gemcitabine $\left(2^{\prime}, 2^{\prime}\right.$-difluoro-2'-deoxycytidine). Can Res 50 (14): 4417-4422, 1990

10. Peters GJ, Bergman AM, Ruiz van Haperen VWT, Veerman G, Kuiper CM, Braakhuis BJ: Interaction between cisplatin and gemcitabine in vitro and in vivo. Semin Oncol 22 supp 11 72-79, 1995

11. Bergman A, Ruiz van Haperen VWT, Weerman G, Kuiper CM, Peters GJ: Synergistic interaction between cisplatin and gemcitabine in vitro. Clin Can Res 2: 521-530, 1996

12. Abbruzzese JL, Frost P: Studies on the mechanism of the synergistic interaction between $2^{\prime}$ deoxy-5-azacytidine and cisplatin. Can Chem Pharm 30: 31-36, 1992

13. Bleiberg H, Conroy T, Paillot B, Lacave AJ, Blijham G, Jacob JH, Bedenne L, Namer M, De Besi P, Gay F, Collette L, Sahmoud T: Randomized phase II study of cisplatin and 5-fluorouracil versus cisplatin alone in advanced squamous cell esophageal cancer. Eur J Cancer 33: 1216-1220, 1997

14. van der Gaast, Kok TC, Kerkhofs L, Siersema PD, Tilanus HW, Splinter TA: Phase I study of a biweekly schedule of a fixed dose of cisplatin with increasing doses of paclitaxel in patients with advanced esophageal cancer. B J Cancer 80: 1052-1057, 1999

15. van der Gaast A, Polee M, Kok TC, Splinter TAW: Phase I study with weekly cisplatin and increasing doses of paclitaxel in patients with esophageal cancer. Proc Am Soc Clin Oncol 18:1162, 1999

16. Ilson DH, Ajani J, Bhalla, Forastiere A, Huang Y, Patel P, Martin L, Donegan J, Pazdur R, Reed C, Kelsen DP: Phase II trial of paclitaxel, fluorouracil, and cisplatin in patients with advanced carcinoma of the esophagus. J Clin Oncol 16: 1826-1834, 1998

17. Ilson DH, Forastiere A, Arquette M, Costa F, Heelan R, Huang Y, Kelsen D: A phase II trial of paclitaxel and cisplatin in patients with advanced carcinoma of the esophagus. Cancer J 6: 316-323, 2000

18. Sandler AB, Kindler HL, Einhorn LH, Mitchell E, Masters G, Kraut M, Nicol S, Raghavan D: Phase II trial of gemcitabine in patients with previously untreated metastatic cancer of the esophagus or gastroesophageal junction. Ann Oncol 11: $1161-1164,2000$

19. Pipp M, Mulkerin D, Warren D, Hotchkis W, Berlin J, Thomas JP: A phase II trial of gemcitabine and 5-fluorouracil in advanced esophageal cancer. Proc Am Soc Clin Oncol 20: 630, 2001

20. Fleming DR, Glisson SD, Bhupalam L, Michelson GD, Goldsmith GH, LaRocca RV: Phase I study of paclitaxel and day $1 /$ day 8 gemcitabine in patients with solid malignancies. Am J Clin Oncol 23: 349-352, 2000

21. Kroep JR, Peters GJ, van Moorsel CJ, Catik A, Vermorken JB, Pinedo HM, van Groeningen CJ: Gemcitabine-cisplatin: A schedule finding study. Ann Oncol 10: 1503-1510, 1999

Address for offprints: Susan G. Urba, University of Michigan, Comprehensive Cancer Center, 1500 E. Medical Center Drive, Room 1366, Box 0922, Ann Arbor, MI 48109-0922; Tel.: (734) 936-5281; fax: (734) 647-8792; Email: surba@umich.edu 